\title{
Características diferenciales de los pacientes con síndrome coronario agudo sin elevación del segmento ST con respecto a los que tienen elevación transitoria del segmento ST
}

\section{E. Arroyo Úcar a ${ }^{a}$ A. Domínguez-Rodríguez ${ }^{a, *}$, R. Juárez Prera ${ }^{a}, G$. Blanco Palacios ${ }^{a}$, C. Hernández García ${ }^{a}$, M. Carrillo-Pérez Tome ${ }^{a}$ y P. Abreu-González ${ }^{\mathrm{b}}$}

a Servicio de Cardiología, Hospital Universitario de Canarias, Tenerife, España

${ }^{\mathrm{b}}$ Departamento de Fisiología, Universidad de La Laguna, Tenerife, España

Recibido el 25 de diciembre de 2010; aceptado el 11 de enero de 2011

Disponible en Internet el 24 de febrero de 2011

\section{PALABRAS CLAVE \\ Síndrome coronario agudo; \\ Elevación transitoria del segmento ST; \\ Manejo terapéutico}

\begin{abstract}
Resumen
Objetivo: Evaluar características diferenciales de los pacientes con síndrome coronario agudo (SCA) sin elevación del segmento ST en comparación con elevación transitoria del segmento ST. Diseño: Estudio prospectivo observacional.

Ámbito: Unidad coronaria de 12 camas.

Pacientes: Enfermos consecutivos de SCA sin elevación persistente del segmento ST.

Principales variables de interés: La población se dividió en 2 grupos, en función de la presencia de elevación transitoria del segmento ST. Las variables de interés fueron: edad, factores de riesgos cardiovasculares, concentraciones de troponina I y glucemia al ingreso, anatomía coronaria, fracción de eyección del ventrículo izquierdo, mortalidad intrahospitalaria y fármacos intrahospitalarios.

Resultados: Los pacientes identificados con SCA y elevación transitoria del segmento ST eran significativamente más jóvenes, fumadores y con predominio del sexo masculino. A su vez, presentaban una elevación pico de troponina I menor, mayor fracción de eyección y principalmente enfermedad coronaria de un vaso.

Conclusiones: Los pacientes con SCA con elevación transitoria del segmento ST presentan diferencias en cuanto al tipo de población, daño miocárdico y resultados angiográficos con respecto a los pacientes con SCA sin elevación del segmento ST. Son necesarias más investigaciones para esclarecer si dichas diferencias implicarían un manejo terapéutico diferente.

(c) 2010 Elsevier España, S.L. y SEMICYUC. Todos los derechos reservados.
\end{abstract}

* Autor para correspondencia.

Correo electrónico: adrvdg@hotmail.com (A. Domínguez-Rodríguez).

0210-5691/\$ - see front matter @ 2010 Elsevier España, S.L. y SEMICYUC. Todos los derechos reservados. doi:10.1016/j.medin.2011.01.007 


\section{KEYWORDS}

Acute coronary syndrome;

Transient ST-segment

elevation;

Therapeutic

management
Differential characteristics of patients with acute coronary syndrome without ST-segment elevation compared to those with transient ST-segment elevation

\begin{abstract}
Objective: To evaluate different characteristics of patients with acute coronary syndrome (ACS) without ST-segment elevation compared with transient St-segment elevation.

Design: An observational, prospective study.

Setting: A 12-bed coronary care unit.

Patients: Consecutive patients of ACS without persistent ST-segment elevation.

Main variables of interest: The population was divided intro 2 groups according to the presence of transient ST-segment elevation. Variables of interest were age, cardiovascular risk factors, troponin I and glucose concentrations on admission, coronary anatomy, left ventricular ejection fraction, inhospital mortality and drugs.

Results: Patients identified as ACS with transient ST-segment elevation were significantly younger, smokers and predominantly male. At the same time, they showed a minor peak elevation of troponin I, a higher ejection fraction and, mainly single-vessel coronary disease.

Conclusions: Patients with ACS with transient ST-segment elevation differ in the type of population, myocardial damage and coronary angiographic results with respect to patients with ACS without ST-segment elevation. More research is needed to clarify whether these differences imply a different therapeutic approach.

(c) 2010 Elsevier España, S.L. and SEMICYUC. All rights reserved.
\end{abstract}

\section{Introducción}

El síndrome coronario agudo (SCA) con elevación no persistente del segmento ST es una entidad clínica caracterizada por la fugacidad de los cambios electrocardiográficos. Se define la elevación transitoria o no persistente del segmento ST como aquella cuya resolución se alcanza en menos de 30 min sin terapia de reperfusión alguna ${ }^{1}$. Las actuales guías terapéuticas indican un manejo similar al del $\mathrm{SCA}^{2}$ sin elevación del segmento ST, no existiendo en número suficiente trabajos o publicaciones que avalen dichas recomendaciones ni que ahonden en las características diferenciales de ambas entidades. Mediante este estudio, se pretende profundizar en dichos aspectos, que consideramos pudieran tener relevancia en su manejo y pronóstico.

\section{Pacientes y métodos}

Estudio de cohorte prospectivo, que abarca desde noviembre de 2008 a octubre de 2009, con seguimiento intrahospitalario, en el que se evaluó a todos los pacientes admitidos en la unidad coronaria de un servicio de cardiología de un hospital terciario con el diagnóstico de SCA sin elevación persistente del segmento ST. Asimismo, se dividió esta población en dos grupos, dependiendo de la existencia o no de elevación transitoria del segmento ST. El diagnóstico de SCA se estableció según los criterios publicados en la literatura ${ }^{3}$ y los pacientes con elevación del segmento ST > $1 \mathrm{~mm}$ en el primer electrocardiograma y resolución en menos de 30 min sin terapia de reperfusión fueron clasificados con SCA con elevación transitoria del segmento ST.

En ambos grupos, se analizaron variables demográficas y clínicas basales (edad, sexo, historia de hipertensión arterial, diabetes mellitus, dislipemia y tabaquismo), analíticas (pico de troponina I, glucemia), hemodinámicas (anatomía coronaria, fracción de eyección) y mortalidad intrahospita- laria. También se analizó en ambos grupos el tratamiento farmacológico recibido durante la hospitalización ${ }^{4}$.

El estudio fue aprobado por el Comité de Ética y el Comité de Ensayos Clínicos e Investigación de la institución y todos los pacientes firmaron el consentimiento informado.

Los datos recogidos fueron analizados mediante el programa estadístico SPSS versión 15.0 para Windows. Las variables cualitativas se expresan como porcentajes, mientras que las cuantitativas, como media \pm desviación estándar. La proximidad a la distribución normal de nuestra población fue evaluada mediante la prueba de KolmogorovSmirnov. Para la comparación entre las variables cualitativas se empleó la prueba de la $\chi^{2}$. La comparación entre variables cuantitativas se realizó mediante la prueba de la t de Student. Se consideró los contrastes de hipótesis bilaterales $\mathrm{y}$ un nivel de significación de $\mathrm{p}<0,05$ en todos los casos.

\section{Resultados}

Una muestra consecutiva de 380 pacientes fue identificada como SCA sin elevación persistente del segmento ST (tabla 1). Todos estos pacientes fueron tratados en igual medida con aspirina, nitroglicerina, clopidogrel, estatinas e inhibidores de la glucoproteína Ilb/Illa. Del total de estos pacientes, a 35 de ellos $(9,2 \%)$ se clasificó como con SCA con elevación transitoria del segmento ST. Estos pacientes presentaban una elevación del segmento ST media de $4 \pm 2,5 \mathrm{~mm}$ y con un tiempo de resolución de los cambios electrocardiográficos de $20 \pm 8 \mathrm{~min}$. Los pacientes identificados con SCA con elevación transitoria del segmento ST eran significativamente más jóvenes, fumadores y con predominio del sexo masculino. Los datos analíticos reflejaron, a su vez, una elevación pico de troponina I menor en este grupo de pacientes. Respecto de los resultados angiográficos coronarios, los pacientes con SCA con elevación transitoria del segmento ST presentaban mayor afectación 
Tabla 1 Características demográficas, analíticas y hemodinámicas de la población de estudio

\begin{tabular}{|c|c|c|c|}
\hline & $\begin{array}{l}\text { SCA sin elevación del segmento } \\
\text { ST }(n=345)\end{array}$ & $\begin{array}{l}\text { SCA con elevación transitoria } \\
\text { del segmento ST }(n=35)\end{array}$ & $\mathrm{p}$ \\
\hline Edad (años) & $64 \pm 18$ & $52 \pm 8$ & 0,001 \\
\hline Varones & $230(67)$ & $35(100)$ & 0,04 \\
\hline Hipertensión arterial & $101(29)$ & $10(28)$ & 0,4 \\
\hline Diabetes mellitus & $95(27)$ & $6(17)$ & 0,84 \\
\hline Hipercolesterolemia & $185(54)$ & $15(43)$ & 0,66 \\
\hline Fumador & $170(49)$ & $35(100)$ & 0,01 \\
\hline Pico de troponina la (ng/dl) & $12 \pm 6,3$ & $8 \pm 4,8$ & 0,003 \\
\hline Glucemia al ingreso (mg/dl) & $148 \pm 68$ & $139 \pm 60$ & 0,45 \\
\hline LONS & $70(20)$ & $4(11)$ & 0,68 \\
\hline Lesión de un vaso & $144(42)$ & $29(83)$ & 0,01 \\
\hline Lesiones de 2 vasos & $101(29)$ & $2(6)$ & 0,06 \\
\hline Lesiones de 3 vasos & $30(9)$ & 0 & 0,8 \\
\hline FEVI & $55 \pm 17$ & $65 \pm 12$ & 0,008 \\
\hline Mortalidad hospitalaria & $4(1)$ & 0 & 0,9 \\
\hline
\end{tabular}

FEVI: fracción de eyección del ventrículo izquierdo; LONS: lesiones obstructivas no significativas; SCA: síndrome coronario agudo. Los valores se expresan como $n$ (\%) o media \pm desviación estándar.

a La muestra de sangre para la determinación de la troponina I se determinó cada $8 \mathrm{~h}$ durante el primer día del ingreso, luego a las 48 , 72 y $96 \mathrm{~h}$. De todas estas determinaciones, se consideró la concentración máxima como el pico de troponina I.

de un solo vaso. La fracción de eyección del ventrículo izquierdo evaluada mediante ecocardiografía transtorácica en las primeras $48 \mathrm{~h}$ del ingreso fue mayor en el grupo de SCA con elevación transitoria del segmento ST. No se encontró diferencias estadísticamente significativas en la mortalidad intrahospitalaria.

\section{Discusión}

En nuestra población de estudio, los pacientes con SCA con elevación transitoria del segmento ST presentan diferencias en cuanto al tipo de población, daño miocárdico y resultados angiográficos con respecto a los pacientes con SCA sin elevación del segmento ST. Constituyen, pues, un subgrupo especial de pacientes con características propias, claramente diferenciales de otros SCA. Dada la escasez de ensayos clínicos previos, queda sin esclarecer si dichas diferencias implicarían un manejo terapéutico diferente, que derivara en un beneficio pleno en dicha población en cuestión ${ }^{5,6}$.

Clásicamente, se considera que la elevación del segmento ST en el electrocardiograma traduce la oclusión completa de un vaso coronario epicárdico ${ }^{7}$, por lo que la fugacidad de dichos cambios implicaría la rápida resolución del evento. Esto, junto con la menor elevación de biomarcadores típicos de daño miocárdico, el predominio de enfermedad de un solo vaso coronario y la mayor fracción de eyección del ventrículo izquierdo, hace plantearnos diferencias fisiopatológicas subyacentes, en las que, en mayor o menor medida, intervengan los fenómenos de aterotrombosis y vasospasmo ${ }^{8}$.

En nuestro estudio, no encontramos diferencias entre ambos grupos respecto a la mortalidad intrahospitalaria; sin embargo, estimamos necesario un seguimiento a largo plazo para establecer diferencias pronósticas ${ }^{9-11}$. Probablemente, el poco interés despertado por este tipo de pacientes y la escasa repercusión bibliográfica residan en la poca impor- tancia y la indiferenciación del SCA con elevación transitoria del segmento ST aplicada por las guías de práctica clínica. Consideramos necesario la realización de más estudios que esclarezcan dicha cuestión y que aporten nuevas ideas en nuestro conocimiento sobre dicha enfermedad.

\section{Conflicto de intereses}

Los autores declaran no tener conflictos de intereses.

\section{Bibliografía}

1. Drew BJ, Pelter MM, Adams MG. Frequency, characteristics, and clinical significance of transient ST segment elevation in patients with acute coronary syndromes. Eur Heart J. 2002;23:941-7.

2. Bertrand ME, Simoons ML, Fox KA, Wallentin LC, Hamm CW, McFadden E, et al. Management of acute coronary syndromes in patients presenting without persistent ST-segment elevation. Eur Heart J. 2002;23:1809-40.

3. Kalra S, Duggal S, Valdez G, Smalligan RD. Review of acute coronary syndrome diagnosis and management. Postgrad Med. 2008;120:18-27.

4. Cohen M, Diez JE, Levine GN, Ferguson 3rd JJ, Morrow DA, Rao SV, et al. Pharmacoinvasive management of acute coronary syndrome: incorporating the 2007 ACC/AHA guidelines: the CATH (cardiac catheterization and antithrombotic therapy in the hospital) Clinical Consensus Panel Report-III. J Invasive Cardiol. 2007;19:525-38.

5. Boersma E, Pieper KS, Steyerberg EW, Wilcox RG, Chang WC, Lee $\mathrm{KL}$, et al. Predictors of outcome in patients with acute coronary syndromes without persistent ST-segment elevation. Results from an international trial of 9461 patients. The PURSUIT Investigators. Circulation. 2000;101:2557-67.

6. Meisel SR, Dagan Y, Blondheim DS, Dacca S, Shochat M, Kazatsker $M$, et al. Transient ST-elevation myocardial infarction: clinical course with intense medical therapy and early invasive approach, and comparison with persistent ST-elevation myocardial infarction. Am Heart J. 2008;155:848-54. 
7. MacDonald RG, Hill JA, Feldman RL. ST segment response to acute coronary occlusion: coronary hemodynamic and angiographic determinants of direction of ST segment shift. Circulation. 1986;74:973-9.

8. Maseri A, Severi S, Nes MD, L'Abbate A, Chierchia S, Marzilli M, et al. "Variant" angina: one aspect of a continuous spectrum of vasospastic myocardial ischemia. Pathogenetic mechanisms, estimated incidence and clinical and coronary arteriographic findings in 138 patients. Am J Cardiol. 1978;42:1019-35.

9. Figueras J, Cortadellas J, Barrabés J, Domingo E, Hermosilla E. Long-term cardiac events in men and women with angina at rest and transient ST segment elevation. Heart. 2007;93:379-80.

10. Yasue $H$, Takizawa A, Nagao $M$, Nishida $S$, Horie $M$, Kubota J, et al. Long-term prognosis for patients with variant angina and influential factors. Circulation. 1988;78: $1-9$.

11. Civeira Murillo E, Del Nogal Saez F, Alvarez Ruiz AP, Ferrero Zorita J, Alcantara AG, Aguado GH, et al. Recomendaciones para el diagnóstico y tratamiento del síndrome coronario agudo sin elevación del segmento ST. Med Intensiva. 2010;34: 22-45. 